\title{
YIG Film for Magnetic Field Sensor
}

\author{
A.O. Kaya, S.Atalay*, H.Gencer, O.A.Kaya, V.S.Kolat, T.IzGi \\ Inonu University Science and Arts Faculty, Physics Department 44069 Malatya, Turkey
}

\begin{abstract}
Single crystal $\mathrm{Y}_{3} \mathrm{Fe}_{5} \mathrm{O}_{12}$ (YIG) film was grown onto (111) oriented gadolinium gallium garnet (GGG) substrate by the liquid phase epitaxy (PLD) technique. The X-ray diffraction measurements showed that epitaxial growth of the film along its (111) axis. The surface characteristic was investigated by atomic force microscopy (AFM) measurement. The magnetic field sensor consisted of a rectangular shape with $5 \mathrm{~mm}$ wide, $15 \mathrm{~mm}$ long and $5 \mu \mathrm{m}$ thick YIG film and a pair of identical $50 \mu \mathrm{m}$ wide microstrip copper transducers elements separated by $6 \mathrm{~mm}$. The filter was tested by measuring reflection $S_{11}$ characteristic at various bias magnetic fields. The results have showed that when the bias field increased from 0 to $2.5 \mathrm{kOe}$, the frequency value corresponding to $\mathrm{S}_{11}$ maxima increased from $1 \mathrm{GHz}$ to $9 \mathrm{GHz}$. This suggests that the wide range magnetic field sensing and the highly sensitive field sensing are simultaneously fulfilled with the YIG film.
\end{abstract}

DOI: $10.12693 /$ APhysPolA.127.937

PACS: 81.15.Gh, 84.40.Az, 75.30.Ds, 41.20.Jb, 85.75.Ss

\section{Introduction}

Recent advances in nanofabrication technology have resulted in the development of novel micro- and nanostructured materials with tunable magnetic properties and submicron- and nano-scale components of magnetic devices. Magnetic nanostructures have great potential for applications in modern technologies such as information storage, biomedicine and microwave and magnetic field sensing. Signal processing devices based on magnetic nanostructures and controlled by magnetic fields provide an opportunity to use magnetostatic spin waves (MSW) as elementary information carriers. Recently, the researchers have shifted to the area of magnetic field controlled devices in which MSW are used to store, carry and process information. This new research field is growing exponentially. MSW have been known since discovered by Damon and Eshbach [1] in 1961 but, they began to be used in the field of high-frequency microwave signal processing devices technology much later, mainly in connection with the discovery of YIG films.

The yttrium iron garnet (YIG) films based on MSW have been researched for decades because of their wide range of applications in the microwave, communication and magnetic detection areas such as delay lines, oscillators, filters, resonators, pulse separators, multi-channel and compressive receivers, core devices in many microwave generators and analyzers, phase shifters and tuners in phased array radar systems and magnetic field sensors [2-4]. The main reason of attraction of YIG films is that the low magnetic loss, narrow ferromagnetic resonance line width, moderate value of saturation magnetization and low coercivity which are favorable for microwave devices [2]. In addition, low damping in YIG films allows spin-wave propagation to be observed over centimeter distances [3].

corresponding author; e-mail: selcuk.atalay@inonu.edu.tr
Recently, it has been recognized that YIG films could be designed as a highly sensitive magnetic field sensor $[4,5]$. Conventional magnetic field sensors have been in use for well hundreds of years. But the technological applications of conventional magnetic field sensors were limited due to the important consideration such as size, weight, sped, performance and cost. Today, the technology for sensing magnetic fields has also evolved driven by the need for improved sensitivity, smaller size, weight, speed, low noise, low cost and high frequency operating range up to gigahertz. In recent years, various different types of magnetic field sensors based on MSW in YIG films and their applications have been investigated. In the literature, many techniques have bee used for preparing YIG films [6,7]. Among them, it is generally accepted that Pulsed Laser Deposition (PLD) technique is a simple and effective way to prepare high quality films of ferrite materials. Up to now, structural and magnetic properties, film quality and technological applications of many YIG films grown by PLD onto $\mathrm{Gd}_{3} \mathrm{Ga}_{5} \mathrm{O}_{12}$ (GGG) substrates were investigated in detail $[8,9]$.

In this study, structural and magnetic properties and magnetic field sensor capacity of single crystal $\mathrm{Y}_{3} \mathrm{Fe}_{5} \mathrm{O}_{12}$ (YIG) film grown by PLD onto $\mathrm{Gd}_{3} \mathrm{Ga}_{5} \mathrm{O}_{12}$ (GGG) substrates were investigated in detail. The designed sensor was tested by measuring reflection characteristic at various bias magnetic fields.

\section{Experimental}

The YIG $\left(\mathrm{Y}_{3} \mathrm{Fe}_{5} \mathrm{O}_{12}\right)$ film was prepared by the pulsed laser deposition (PLD) technique onto gadolinium gallium garnet $(\mathrm{GGG})$ substrate. The deposited film thickness was about $5 \mu \mathrm{m}$. The back side of the substrate was metalized and grounded. The magnetic field sensor consist of a rectangular shape with $5 \mathrm{~mm}$ wide, $15 \mathrm{~mm}$ long and $5 \mu \mathrm{m}$ thick YIG film and a pair of identical $50 \mu \mathrm{m}$ wide microstrip cooper transducers elements separated by $6 \mathrm{~mm}$. The two ports of the transducers were connected to a vector network analyzer, which generates 
the microwave excitation at one port and measures the transmission at the other port. A pair of electromagnets was used to generate the bias magnetic field parallel to the film surface and perpendicular to the direction of magnetostatic wave propagation. The magnetic sensor was tested by measuring $S_{11}$ reflection characteristic at various magnetic fields. In $S_{11}$ measurements as a function of magnetic field, the $S_{11}$ signal was normalized to the $\mathrm{S}_{11}$ signal measured at $\mathrm{H}=0$ Oe.

\section{Results and Discussion}

$\mathrm{X}$-ray diffraction measurement was used to investigate the structural property of the YIG film grown on to GGG substrate. X-ray diffraction spectrum showed that the growth was along the (111) direction. Nearly overlapped YIG (888) and GGG (888) peaks have showed that the lattice parameters of YIG film and GGG substrate are very close to each other. The surface morphology and roughness of the film have been studied by atomic force microscopy (AFM). Figure 1 shows the AFM images for single crystal $\mathrm{Y}_{3} \mathrm{Fe}_{5} \mathrm{O}_{12}$ film was grown onto $\mathrm{Gd}_{3} \mathrm{Ga}_{5} \mathrm{O}_{12}$ substrate by PLD technique with a thickness of $5 \mu \mathrm{m}$. The surface morphology and roughness have been measured over 10 and $5 \mu^{2}$ range (Fig. $1 \mathrm{a}$ and b). The results have showed that the surface condition is smooth and the size of the grains is uniform. The root mean square (rms) value of the surface roughness is $1.4 \mathrm{~nm}$ which is in agreement with the literature values ranging from 0.2 to $7 \mathrm{~nm}[10]$.
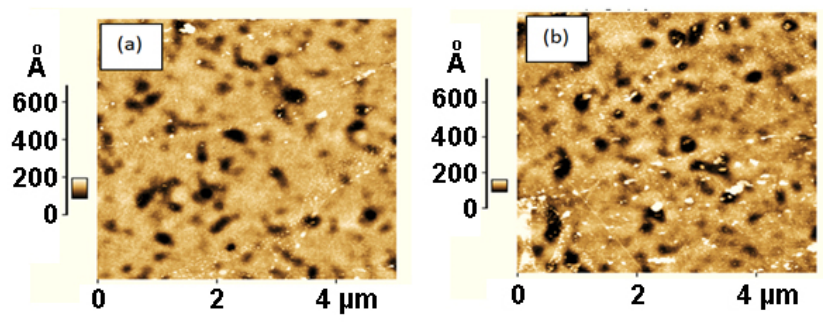

Fig. 1. AFM images taken from center (a) and edge (b) of the YIG film grown onto GGG substrate.

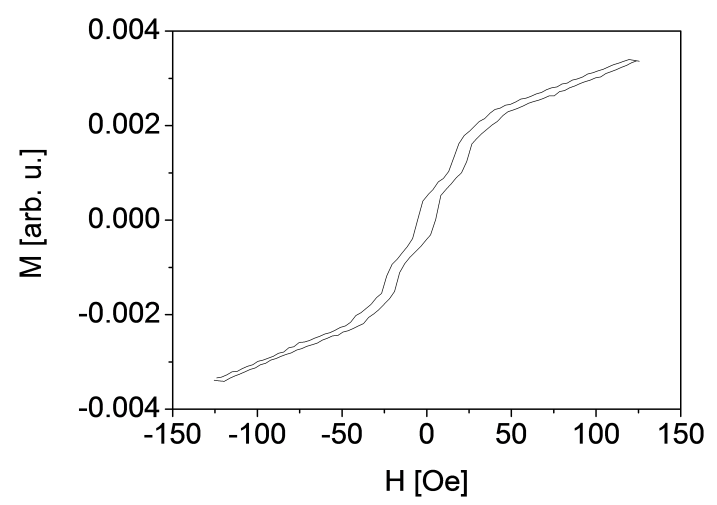

Fig. 2. Hysteresis loop for YIG film grown onto GGG substrate.
Figure 2 shows in-plane magnetization curve for PLD deposited YIG film measured with a vibrating sample magnetometer (VSM). Previous studies have showed that the coercive field is a good indicator of the quality of PLD made YIG films as a planar waveguide $[4,5]$. The coercive field was found to be 5.5 Oe which is small enough for prepared YIG film to use as a waveguide. However, as can be seen in Fig. 2, a step-like magnetization behavior was observed in hysteresis loop. The shape of hysteresis loop is in agreement with that of the liquid phase epitaxy (LPE) deposited YIG films [11]. Shkar et al. [11] have concluded that the step-like behavior of magnetization curve of the film is due to the domain walls moving stepwise with increasing external magnetic field. In the absence of an applied magnetic field, a ferrite specimen will subdivide into many small domains in which the direction of the moments alternates. By applying a DC magnetic field the domains begins to rotate towards to field direction. Due to the strong domain wall interaction, the rotation of domains is not continuous with increasing magnetic field. As a consequence, a step-like behavior in magnetization loop was observed.

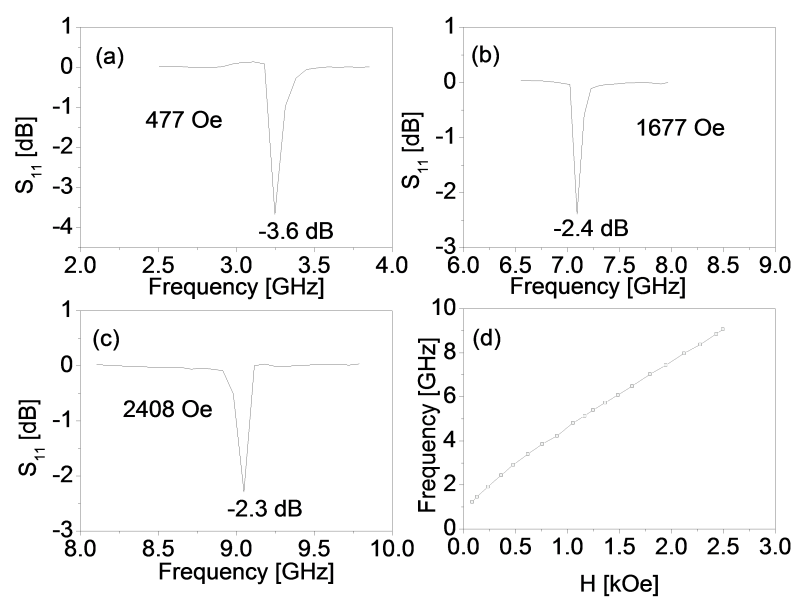

Fig. 3. The variation of return loss $\left(\mathrm{S}_{11}\right)$ and the resonant frequency with applied magnetic field.

As discussed above, the sensing element of the proposed sensor is YIG film grown onto (111) oriented gadolinium gallium garnet (GGG) substrate by PLD technique. The back side of the substrate was metalized and grounded. The magnetic field sensor consist of a rectangular shape with $5 \mathrm{~mm}$ wide, $15 \mathrm{~mm}$ long and $5 \mu \mathrm{m}$ thick YIG film and a pair of identical $50 \mu \mathrm{m}$ wide microstrip cooper transducers elements separated by $6 \mathrm{~mm}$. The two ports of the transducers were connected to a vector network analyzer, which generates the microwave excitation at one port and measures the transmission at the other port. In order to excite magnetostatic surface wave (MSSW), the biasing magnetic field from an external electromagnet was applied parallel to the film surface and perpendicular to the direction of magnetostatic wave propagation. The magnetic sensor was tested by measuring reflection $\mathrm{S}_{11}$ (return loss) characteristic at various magnetic fields. The measured return loss $\left(S_{11}\right)$ charac- 
teristics are shown in Fig. 3a-c for 477, 1677 and 2408 Oe magnetic fields respectively. The measured $\mathrm{S}_{11}$ values are $-3.6,-24$ and $-2.3 \mathrm{~dB}$ for 477,1677 and 2408 Oe magnetic fields, respectively. The variation of peak frequency in a whole range of external magnetic field was given in Fig. 3d. As can be seen, when the bias field increased from 0 to $2.5 \mathrm{kOe}$, peak frequency increased from nearly $1 \mathrm{GHz}$ to $9 \mathrm{GHz}$. This suggests that the wide range magnetic field ( 0 to $2.5 \mathrm{kOe}$ ) sensing and the highly sensitive field sensing simultaneously fulfilled with the YIG film.

\section{Conclusions}

In order to excite magnetostatic surface wave (MSSW), the biasing magnetic field was applied parallel to the film surface and perpendicular to the direction of magnetostatic wave propagation. The filter was tested by measuring reflection $\mathrm{S}_{11}$ characteristic at various bias magnetic fields. The results have showed that when the bias field increased from 0 to $2.5 \mathrm{kOe}$, the frequency value corresponding to $\mathrm{S}_{11}$ maxima increased from $1 \mathrm{GHz}$ to $9 \mathrm{GHz}$. This suggests that the wide range magnetic field sensing and the highly sensitive field sensing are simultaneously fulfilled with the YIG film

\section{Acknowledgments}

This work was supported by TUBİTAK with project number $112 \mathrm{~T} 820$.

\section{References}

[1] R.W. Damon, J.R. Eshbach, J. Phys. Chem. Solids 19, 308 (1961).

[2] Y.F. Chen, K.T. Wu, T.D. Yao, C.H. Peng, K.L. You, W.S. Tse, Microelectronic Engineering 81, 329 (2005).

[3] A.A. Serga, A.V. Chumk, B. Hillebrands, J. Phys. D: Appl.Phys. 43, 264002 (2010).

[4] O. Kamada, H. Minemoto, S. Ishizuka, J. Appl. Phys. 61, 3268 (1987).

[5] C. Holthaus, I. Nistor, I.D. Mayergoyz, C. Krafft, J. Appl. Phys. 99, 08B308 (2006).

[6] M. Huang, Z.C. Xu, Thin Solids Films 450, 324 (2004).

[7] M. Kucera, J. Bok, K. Nitsch, Solid State Commun. 69, 1117 (1989).

[8] N. Kumar, D.S. Misra, N. Venkataramani, S. Prasad, R. Krisnnan, J. Magn. Magn. Mater. 272, E899 (2004).

[9] S. Higuchi, K. Ueda, F. Yahiro, Y. Nakata, H. Uetsuhara, T. Okada, M. Maeda, IEEE Trans. Magn. 37, 2451 (2001).

[10] Y. Sun, Y.Y. Song, M. Wu, Appl. Phys. Lett. 101 082405 (2012).

[11] V.F. Shkar, E.I. Nikolaev, V.N. Sayapin, A.I. Linnik, V.P. Denysenkov, A.M. Grishin, S.I. Khartsev, Physics of the solid State 47, 1071 (2005). 